\title{
Verbetes Oral, Oralidade e Discurso: Análise Crítica de Dois Dicionários Didáticos de Ensino de Línguas Estrangeiras/Oral
}

\author{
Orality and Discourse Entries: A Critical Analysis of Two \\ Foreign Language Teaching Dictionaries
}

\section{Maria da Glória Magalhães dos REIS* Alice Maria Araújo FERREIRA**}

Resumo: Este artigo tem como objetivo apresentar uma pesquisa que vem sendo desenvolvida pelo GPTT (Grupo de Pesquisa em Terminologia e Tradução) e que se propõe a realizar uma análise crítico-comparativa de dois dicionários: Galisson e Coste (1976) e Cuq (2003). Neste trabalho, especificamente, abordamos o verbete “oral”. Para a realização da análise serão usadas como fundamentação teórica outras obras dos autores em questão Cuq (2005) e Galisson e Puren (1999) e de autores que se debruçaram sobre a questão da subjetividade (CORACINI, 1999, 2005; CORACINI; GHIRALDELO, 2011), do discurso (BAKHTIN, 2010, 2010; SCHNEUWLY; DOLZ, 2010) e da oralidade (MESCHONNIC, 1982, 1999; MARCUSCHI, 2008, 2010). Observa-se, através deste trabalho, como a questão da oralidade, essencial nas discussões sobre ensino de línguas na atualidade, precisa ainda ser explorada tanto no domínio da Didática de Línguas e Culturas, de linha francesa, quanto no domínio da Linguística Aplicada, no Brasil, levando em conta seus aspectos subjetivos e discursivos.

\footnotetext{
${ }^{1}$ Doutorado em Língua e Literatura Francesa pela Universidade de São Paulo (2008). Professora Doutora Adjunta 2 - Universidade de Brasília (UnB). Departamento de Línguas Estrangeiras e Tradução - LET. Programa de Pós-Graduação em Linguística Aplicada - PPGLA. Contato: gloriamagalhaes@gmail.com.

${ }^{2}$ Doutorado em Linguística pela Universidade de São Paulo (2000). Professora Doutora Adjunta 2 - Universidade de Brasília (UnB). Departamento de Línguas Estrangeiras e Tradução - LET. Programa Pós-Graduação em Tradução - POSTRAD. Contato: alicemaf@yahoo.com.br.
} 
Palavras-chave: Oral. Dicionário. Ensino de Língua Estrangeira.

\begin{abstract}
This article aims at presenting a research developed by GPTT (Research group of terminology and translation) that intends to undertake a critical-comparative analysis of two dictionaries: Coste \& Galisson (1976) and Cuq (2003). Specifically here, we discuss the entry "oral". To carry on the analysis, we refer to other works by the authors already mentioned among others, such as Cuq (2005) and Galisson and Puren (1999), as well as authors interested in approach the issue of subjectivity (CORACINI, 1999, 2005; CORACINI; GHIRALDELO, 2011), discourse (BAKHTIN, 2010, 2010; SCHNEUWLY; DOLZ, 2010), and orality (MESCHONNIC, 1982, 1999; MARCUSCHI, 2008, 2010). The analysis has showed that orality constitutes an essential issue in the discussion about teaching languages nowadays, thus demanding being investigated in the French domain of Languages and Culture Didactics, as well as in Applied Linguistics, in Brazil, considering subjective and discursive aspects.
\end{abstract}

Key-words: Oral. Dictionary. Foreign Language.

\title{
Introdução
}

A pesquisa descrita neste artigo envolve duas áreas do Departamento de Línguas Estrangeiras e Tradução da Universidade de Brasília: Linguística Aplicada e Tradução e se propõe a analisar dois dicionários de didática de língua estrangeira de linha francesa. Os dois dicionários analisados são Dictionnaire de didactique des langues (1976) (doravante DDL), cuja organização é compartilhada entre Robert Galisson e Daniel Coste e Dictionnaire de didactique du français langue étrangère et seconde (2003), organizado por Jean-Pierre Cuq (doravante DDFLES).

O estudo visa a contribuir para o registro histórico e crítico da trajetória de um domínio de estudo que, na França, passou a ser chamado de "Didactique des Langues et des Cultures" [Didática das Línguas e Culturas]. Robert Galisson, em seu livro Formation en questions, propõe também a denominação "Didactologie des Langues et des Cultures" [Didactologia das Línguas e das Culturas], e a descreve como

Disciplina de intervenção prioritariamente focada nos atores de campo, cujo objetivo é trabalhar para a otimização do processo de contrução- 
transmissão dos saberes e dos savoir-faire na área da educação às línguas-culturas. (GALISSON; PUREN, 1999, p. 118, tradução nossa) ${ }^{1}$.

Tal nome não foi adotado de forma global na França, firmando-se a disciplina como "Didactiques de Langues et des Cultures". Para Cuq (2005, p. 43-74), em histórico da área na obra Cours de didactique du français langue étrangère et seconde, a discussão é complexa e envolve elementos epistemológicos, políticos, pedagógicos e éticos. No entanto, na conclusão do capítulo, o autor opta pelo nome mais específico de "Didática do Francês Língua Estrangeira e Segunda", também parte do título do dicionário organizado por ele.

No Brasil, esses estudos desenvolvem-se, em grande parte das universidades brasileiras, no âmbito da Linguística Aplicada, daí a importância da análise das semelhanças e diferenças dos termos usados nas áreas de Didática das Línguas e Culturas e da Linguística Aplicada com o objetivo de contribuir para o desenvolvimento das duas disciplinas e para a problemática do ensino de línguas em geral e estrangeiras em particular.

No presente trabalho apresentamos, em um primeiro momento, o conceito de "oral" presente nos dicionários analisando-os a partir da perspectiva discursiva e, decorrente dela, das questões ligadas à subjetividade / intersubjetividade e à compreensão da língua como fenômeno social fundado na interação verbal, e da linguagem considerada como ideológica, histórica e condicionada por fatores sociológicos. Por fim, desenvolvemos o conceito de oralidade, ausente como verbete nos dicionários, a partir da concepção de linguagem de Henri Meschonnic $(1982,1999)$ e de Luiz Antonio Marcuschi (2008, 2010).

\section{A Questão do Oral para Galisson e Coste (1976)}

Após o conceito de Competência Comunicativa (ou de comunicação) proposto por Dell Hymes, em 1964, a didática de línguas tem se estabelecido como área interdisciplinar. Na Linguística Aplicada, as reflexões chegam a propostas de estudos em "campos híbridos" (KLEIN, 1990), ou ainda, para Moita

${ }^{1}$ « discipline d'intervention prioritairement ciblée sur les acteur de terrain, dont l'objectif est d'oeuvrer à l'optimisation du processus de contruction-transmission des savoirs et des savoir-faire en matière d'éducation aux langues-cultures. » 
Lopes (2006, p. 99): “vivemos tempos de hibridismo teórico e metodológico nas ciências sociais e humanas, o que tem tornado as fronteiras disciplinares tênues e sutis". Nesse sentido, o diálogo com a Sociolinguística, a Antropologia Cultural, a Tradução, a Sociologia, a Psicologia, a Teoria da Comunicação e a Ciência da Educação, faz-se imprescindível pelo conhecimento e pelos conceitos que esses domínios fornecem. Essa interação, além de promover outras perspectivas e/ou abordagens à didática de línguas, oferece-lhe ferramentas importantes para a compreensão da língua enquanto prática social e lugar de encontros (inter)culturais.

Uma perspectiva que problematiza o ensino de línguas estrangeiras é trazida pelos estudos sobre o discurso e a psicanálise (CORACINI, 1995; CORACINI; GHIRALDELO, 2011), considerando-se a "constitutividade heterogênea, polifônica do sujeito e do discurso" (CORACINI, 1999, p. 11) e que "todo o indivíduo é constituído e determinado pelo seu inconsciente e que, por isso mesmo, não se pode prever ou predeterminar como vai ser a apreensão de qualquer informação" (CORACINI; GHIRALDELO, 2011, p. 25). Nesse sentido, não se mostra possível preconizar um estudo que aborde o oral e a oralidade sem considerar as várias vozes que perpassam o sujeito aprendente de língua estrangeira, sejam essas vozes reflexos da língua materna ou de outra ou outras línguas estrangeiras que fazem parte de seu universo. Como, então, conceber a noção de oral desvinculada da noção do sujeito sendo que toda a linguagem, que é a via de manifestação do inconsciente, implica na presença de outros em seu dizer? Como não levar em conta a pluralidade das vozes do sujeito que é "habitado por outros" (CORACINI, 2003, p. 150)?

Ainda outra perspectiva que vem sendo abordada em nossas pesquisas é a dos gêneros orais desenvolvida por Bernard Schneuwly e Joaquim Dolz, cujos estudos, na Suíça, se voltam para o ensino-aprendizagem de língua materna. Esta posição é explicitada pelas organizadoras do livro com artigos dos autores "Gêneros orais e escritos na escola" (2010), Roxane Rojo e Glaís Sales Cordeiro, que consideram a importância da noção de gêneros como instrumento para o ensino tanto de leitura e escrita quanto no desenvolvimento da oralidade. Schneuwly e Dolz não concebem a noção apenas de "oral", mas de "orais". Para os autores

... não existe "o oral", mas "os orais" em múltiplas formas, que, por outro lado, entram em relação com os escritos de maneiras muito 
diversas: podem se aproximar da escrita e mesmo dela depender como é o caso da exposição oral ou, ainda mais, do teatro e da leitura para os outros -, como também podem estar mais distanciados - como nos debates ou, é claro, na conversação cotidiana. (SCHNEUWLY; DOLZ, 2010, p. 114).

Essas reflexões inspiram uma concepção muito mais ampla da questão do oral do que a apresentada pelos dicionários analisados. Sendo assim, a partir dessas diferentes perspectivas, passamos à análise e discussão das noções de oral nos dois dicionários. No DDL, Galisson e Coste desenvolvem, em um primeiro momento, o conceito código oral em oposição a código escrito ou língua escrita. Para isso, os autores explicam que "a lingüística do fim do século XIX e do século XX e a metodologia do ensino de línguas sublinharam a distinção entre oral e escrito, situando-a em diferentes níveis"’2 (GALISSON; COSTE, 1976, p. 386). Um primeiro nível sendo o do sistema, do código no qual as duas modalidades, oral e escrito, não podem se confundir, pois há diferenças em relação à própria organização de significantes e, diacronicamente, para o francês, código oral e escrito não evoluíram da mesma forma. Um exemplo desse fato, para os autores, é que: "as marcas de gênero e de número em francês não têm nem a mesma natureza nem a mesma distribuição no oral e no escrito"3 (GALISSON; COSTE, 1976, p. 386).

Um segundo nível, seria o do discurso. Nele, Galisson e Coste tratam, em primeiro lugar, a questão das condições de comunicação no oral e no escrito que são sensivelmente diferentes na medida em que o oral não permite voltar atrás, com tudo o que isso compreende, por exemplo, a produção de lapsos, de hesitações, de rupturas de construção etc., diferentemente do escrito que, de acordo com o autor, “autoriza o remorso". Nesse mesmo nível, o oral dispõe, em compensação, de meios linguísticos, paralinguísticos, extralinguísticos como a entonação, as mímicas, os gestos etc.. Para concluir

2 «La linguistique de la fin du XIX ${ }^{\mathrm{e}}$ siècle et $\mathrm{XX}^{\mathrm{e}}$ siècle et la méthodologie de l'enseignement des langues ont souligné la distinction entre oral et écrit en la situant à différents niveaux. » As traduções dos trechos dos verbetes dos dicionários são de minha autoria.

${ }^{3}$ « les marques de genre et de nombre en français n’ont ni la même nature ni la même distritution à l'oral et à l'écrit. » 
esta fase da definição, os autores afirmam que o oral e o escrito não possuem o mesmo estatuto sociolinguístico: o escrito é considerado mais regrado, mais "culto" e o oral mais afetivo ou espontâneo. Galisson e Coste (1976) acrescentam, contudo, um nível que consideram ter passado por uma análise mais fina e que abole a oposição entre "discurso oral homogêneo" e "discurso escrito homogêneo", tendo em vista que "todo discurso, fenômeno de fala, resulta de diversas variáveis (dizendo respeito ao destinador, ao destinatário, à relação entre eles, ao referente, à situação tanto quanto ao canal de comunicação utilizado)" "(GALISSON; COSTE, 1976, p. 386). Essa multiplicidade de elementos leva a uma multiplicidade de discursos, o que corrobora com a afirmação de Schneuwly e Dolz (2010) da existência de diversos "orais", ou seja, múltiplos discursos orais.

Galisson e Coste (1976) fazem ainda algumas observações sobre a noção de oral. A primeira serve para estabelecer as particularidades dos termos "oral", "oralisado" [oralisé] e "falado" [parlé]. O oral seria um tipo de discurso sem interação real (conferências, comentários radiofônicos, etc.), o oralisado seria um discurso escrito dito em voz alta; já o falado corresponderia a uma comunicação entre interlocutores. A segunda observação do autor desenvolve-se a partir da afirmação de que: com o desenvolvimento das técnicas e das mídias, o discurso "oralisado" pode ser tão preparado quanto o discurso escrito. A terceira diz respeito à tendência atual, na didática de línguas, de ultrapassar a dicotomia oral-escrito. Proposta que, na época do dicionário de Galisson e Coste, ainda estava em seu início e que, hoje, é amplamente divulgada nos trabalhos mais recentes que se voltam para interações contínuas entre oral e escrito (PIETRAROIA, 2000; CUQ, 2005; MASSARO, 2007; REIS, 2008) no ensino de línguas estrangeiras. Também na linguística geral, Luiz Antonio Marcuschi (2008, p. 72) considera a oralidade e a escrita como duas modalidades discursivas, de forma alguma situadas em oposição, mas "igualmente relevantes e fundamentais", cujas diferenças se situam em suas respectivas práticas sociais de produção.

Conclui-se, então, que ainda que a linguagem oral seja diferente da linguagem escrita e que os processos implicados na compreensão e na produção sejam diferentes, prefere-se abordá-los atualmente de forma

\footnotetext{
4 « Tout discours, phénomème de la parole, résulte de diverses variables (tenant au destinateur, au destinatire, à leur relation, au référent, à la situation aussi bien qu'au canal de communication utilisé). »
} 
dialética por meio de atividades que possam estabelecer intersecções entre o oral e o escrito, como descreve Pietraróia (2000, p. 24):

... pude efetivamente constatar uma melhora na performance de leitura dos alunos de FLE que foram levados a se interessar mais pelas especificidades do código escrito e pelas particularidades da língua alvo, o que nos mostra, finalmente, que a aula de leitura deve e pode acolher atividades de saem do campo do escrito para trabalhar a linguagem, com suas interações e representações. Assim, no nível dos conhecimentos lingüísticos, por exemplo, a aprendizagem do código se torna imperativa, mas não se é obrigado a trabalhar apenas sobre o código escrito, [...] daí vem o interesse de motivar os alunos a atividades orais, como a leitura em voz alta e o trabalho com as correspondências grafo-morfológicas. Pode-se pedir também que gravem suas próprias leituras e que as transcrevam para observar os desvios cometidos; pode-se ainda fazer um trabalho com as rimas, a partir de cantigas de roda e de canções $[. ..] .^{5}$

Atividades que motivem o trabalho sobre o aspecto musical e entonativo da língua e aquelas que, como salienta Paulo Massaro (2007, p. 179), proporcionam a "construção de passarelas entre a esfera da escrita e a esfera da oralidade", possibilitam a realização de um caminho que marca o locutor além do puramente linguístico. Tanto na Didática das Línguas e Culturas quanto na Linguística Aplicada, tanto no ensino de língua materna quanto no de língua estrangeira, o trabalho sobre os gêneros orais dependem da concepção do que é o oral para o pesquisador, o formador, o professor e até mesmo do aprendente.

${ }^{5}$ «... j’ai pu effectivement constater une amélioration dans la performance de lecture des étudiants de FLE qui on été poussés à s'intéresser davantage aux spécificités du code écrit et aux particularités de la langue cible, ce qui nous montre, finalement, que la classe de lecture peut et doit accueillir des activités qui sortent du champ de l'écrit pour travailler le langage, avec ses interactions et ses représentations. Ainsi, au niveau des connaissances linguistiques, par exemple, l'intérêt de motiver les étudiants à des activités orales, comme la lecture à haute voix et le travail sur les correspondances grapho-phonologiques. On peut leur demander aussi d'enregistrer leurs propres lectures et de les transcrire pour observer les déviations commises ; on peut encore faire un travail sur les rimes, à partir de comptines et de chansons ... » 
O oral pode ser visto pelo professor, segundo relatos de Schneuwly e Dolz (2010, p. 111) sobre pesquisas desenvolvidas na Suíça, como: materialidade, espontaneidade ou como norma. O autor explica que o oral pode estar ligado tanto a uma "alegria de expressão" quanto ao

... exercício sistemático e de correção que apontam para a visão de língua como norma para a dependência do oral em relação à norma escrita. Mas, igualmente, o oral é visto como lugar privilegiado de espontaneidade e da liberação. (SCHNEUWLY; DOLZ, 2010, p. 112).

Depreende-se dessa afirmação uma concepção mais complexa do oral e um enfoque mais dialético das relações entre o oral e o escrito. Essa concepção compreende também elementos que dizem respeito à subjetividade, o que pode ser observado pelas expressões: "alegria da expressão", "espontaneidade" e "liberação". A subjetividade sendo considerada na epistemologia ocidental como oposta à objetividade (CORACINI; GHIRALDELO, 2011, p. 12), a primeira mostra-se muito frequentemente excluída da discussão tanto da Linguística Aplicada quanto da Didática das Línguas como se fosse possível manter a supremacia de uma em detrimento da outra, ignorando assim o aspecto psicanalítico da fala (manifestação tanto escrita quanto oral). Para Bakhtin (2010, p. 289), o elemento expressivo seria "a relação subjetiva emocionalmente valorativa do falante com o conteúdo do objeto e do sentido do seu enunciado", que é o que determina sua composição e estilo. Mais uma vez observa-se a relação intrínseca entre o oral, a expressividade e a subjetividade.

\section{A Questão do Oral para Cuq (2003)}

Abordamos, em seguida, a definição dada pelo DDFLES, organizado por Jean-Pierre Cuq (2003). Em sua definição de oral, o dicionário traz também, como em Galisson e Coste (1976), a questão da dicotomia entre oral e escrito citando igualmente a irreversibilidade e o imediatismo do processo no oral, em contraste com a comunicação adiada, postergada e a possibilidade de retomada do escrito. Assim como em Galisson e Coste (1976), Cuq (2003), no que diz respeito à relação do escrito como registro de norma culta e do oral ao da familiaridade, chega à conclusão que essa 
separação não tem mais um peso importante na didática de línguas estrangeiras atual.

Cuq (2003) se serve do conceito de "oral" para desenvolver um pequeno histórico da evolução da própria disciplina Didática das Línguas e Culturas e da relação que se estabeleceu, durante esse processo, entre o "oral" e o "escrito", partindo da dicotomia já relatada por Galisson e Coste (1976), chegando a uma relação dialética entre os dois, observando que, por exemplo, a forma oral de uma conferência terá o mesmo nível de elaboração do escrito, ou ainda, que um diálogo de romance pode ter, aparentemente, a mesma espontaneidade do oral. Nesse histórico, o autor parte das metodologias áudio-orais, inspiradas no distribucionismo passando pelos Atos de Fala, chegando à Abordagem Comunicativa. Um aspecto relevante da definição é a observação de que

A ênfase se desloca para outra distinção: o fato de que o oral é tanto um meio de ensino e de aprendizagem, quanto um objetivo em si mesmo. Essa distinção pode se revelar útil por apreciar a evolução do componente oral na história da didática do FLE. ${ }^{6}$ (CUQ, 2003, p. 182).

Se, por um lado, o DDFLES, avança em relação ao DDL ao vislumbrar a língua como meio de ensino e, ao mesmo tempo, objeto de aprendizagem e ao afirmar a importância do aprendizado dos "gêneros orais (explicativos, narrativos, argumentativos, etc.)"7, demonstrando certa imprecisão entre o conceito de gêneros e tipos textuais. Por outro lado, não se remete, em nenhum momento, à questão da subjetividade, enfatizando o uso da língua para a comunicação que ocorre dentro da sala de aula, apresentando uma visão utilitarista da língua em detrimento das questões intersubjetivas.

${ }^{6}$ «L'accent se déplace vers une autre distinction : le fait que l'oral est tantôt un moyen d'enseignement et d'apprentissge, tantôt un objectif à part entière. Cette distinction peut se révéler utile poir apprécier l'évolution de la composante orale dans l’histoire de la didactique du FLE.»

${ }^{7}$ « genres oraux (explicatifs, narratifs, argumentatifs, etc.).» 


\section{A Oralidade para Meschonnic $(1984,1999)$}

$\mathrm{Na}$ abordagem dos dicionários, de um lado, Galisson e Coste (1976) acentuam as concepções da linguística geral, por outro lado, Cuq (2003) valoriza sobretudo a evolução dos métodos de ensino de línguas chegando a novas pesquisas desenvolvidas em torno da intercompreensão de línguas românicas. Cuq (2003) emprega, também, no verbete "oral”, a palavra "oralidade" afirmando que "a concepção da oralidade se enriquece: a abordagem morfossintática é integrada a uma visão mais ampla recorrendo a todos os aspectos não verbais, à etnografia da comunicação e à pragmática"8 (CUQ, 2003, p. 183).

Essa noção de oralidade, que não faz objeto de uma entrada específica em nenhum dos dois autores, parece-nos essencial, atualmente, em relação à tendência, já citada, de tentar abordar o oral e o escrito de uma forma dialética. Nesse sentido, poderíamos citar a definição de oralidade proposta por Henri Meschonnic $(1984,1999)$ na qual os conceitos de discurso, subjetividade e ritmo não podem ser compreendidos separadamente. $\mathrm{Na}$ aprendizagem de uma língua estrangeira, esses elementos interligados desempenham um papel preponderante na forma de vislumbrar o discurso como uma organização do sentido pelo sujeito através do ritmo, em outros termos, a apropriação da oralidade passa a ser o encontro com uma linguagem que atravessa o sujeito e que se dá por um discurso que é organizado por um ritmo. Meschonnic (1999), em Poétique du traduire, vê nos conceitos de língua e discurso a transformação pela qual passou o pensamento sobre a linguagem. Para o autor, "Ele [o pensamento sobre a linguagem] passou da língua (com suas categorias - léxico, morfologia, sintaxe) ao discurso, ao sujeito agindo, dialogando, inscrito prosodicamente, ritmicamente na linguagem"9 (MESCHONNIC, 1999, p.13).

Mesmo que o autor trate da oralidade no contexto da prática da tradução, o texto não deixa de ser, para ele, a enunciação de um sujeito.

${ }^{8}$ « la conception de l'oralité s'enrichit : l'approche morphosyntaxique est intégrée à une vision plus large faisant appel à tous les aspects non verbaux, à l'ethnographie de la communication et à la pragmatique. »

${ }^{9}$ "Elle [la pensée du langage ] est passée de la langue (avec ses catégories - lexique, morphologie, syntaxe) au discours, au sujet agissant, dialoguant, inscrit prosodiquement, rythmiquement dans le langage. » 
Pode-se, portanto, retomar seus conceitos dentro da relação que estabelecemos com a linguagem e a língua e de como as concebemos seja no aprendizado da língua estrangeira, seja na tradução. E, no ensino de línguas estrangeiras, que é o âmbito do presente trabalho, a oralidade dos diferentes discursos pode ser abordada no processo de "inscrição do sujeito na discursividade nova da segunda língua" (CORACINI, 2003, p. 152) que o aprendente deve experimentar e que pode levá-lo ao confronto com sua própria discursividade.

A oralidade, então, passa a ser vislumbrada como estreitamente ligada à subjetividade e ao ritmo, tomando-se, no âmbito de nosso trabalho, a definição de ritmo tal como enunciada por Meschonnic (1982): como movimento de enunciação e como marca da subjetividade, mais explicitamente...

Se o sentido é uma atividade do sujeito, se o ritmo é uma organização do sentido no discurso, o ritmo é necessariamente uma organização ou configuração do sujeito em seu discurso. Uma teoria do ritmo no discurso é portanto uma teoria do sujeito na linguagem. Pode haver teoria do ritmo sem teoria do sujeito, não teoria do sujeito sem teoria do ritmo. A linguagem é um elemento do sujeito, o elemento mais subjetivo, do qual o mais subjetivo por sua vez é o ritmo. (MESCHONNIC, 1982, p. 71). ${ }^{10}$

Se para Meschonnic, os conceitos de discurso, subjetividade e ritmo, como já dissemos, não podem ser separados, na aprendizagem de língua estrangeira essas três noções têm um papel preponderante na forma de vislumbrar a linguagem tal como descrita pelo autor, como uma ação do sujeito que organiza o sentido pelo ritmo. O processo de aprendizagem de uma língua, a sua própria ou a estrangeira, dentro da Didática de Línguas e Culturas ou dentro da Linguística Aplicada não pode ser visto como um

\footnotetext{
${ }^{10}$ « Si le sens est une activité du sujet, si le rythme est une organisation du sens dans le discours, le rythme est nécessairement une organisation ou configuration du sujet dans son discours. Une théorie du rythme dans le discours est donc une théorie du sujet dans le langage. Il ne peut y avoir de théorie du rythme sans théorie du sujet, pas de théorie du sujet sans théorie du rythme. Le langage est un élément du sujet, l'élément le plus subjectif, dont le plus subjectif à son tour est le rythme. »
} 
simples treino de habilidades linguísticas, ou como uma prática de um código. Trata-se, sim, de um encontro com uma nova linguagem na qual a intersubjetividade configura-se como interculturalidade na medida em que o sujeito é levado a construir seu discurso na discursividade do outro.

Essa inscrição do sujeito na linguagem determina a oralidade do seu discurso, o que implica no apagamento da dualidade forma/sentido. Essa oralidade incorpora-se ao processo de individuação, ou seja, do subjetivo na linguagem, da "alegria da expressão", descrita anteriormente por Schneuwly e Dolz (2010), e mostra-se através da sua prosódia, ritmo e significância. Como afirma o teatrólogo Artaud:

... essa faculdade que têm as palavras de criar também elas uma música seguindo a forma pela qual são pronunciadas, independentemente de seu sentido concreto, e que pode mesmo ir contra esse sentido, de criar sob a linguagem uma corrente subterrânea de impressões, de correspondências, de analogias. (ARTAUD, 1964, p. 46).

Dentro de tal concepção, não parece possível eliminar do discurso a ideia da musicalidade e do ritmo que são elementos que atingem a sensibilidade e o inconsciente do indivíduo e o acesso a processos subjetivos que contribuem para o desenvolvimento da expressão. Igualmente não se pode eliminar a ideia dos gêneros orais como práticas sociais historicamente situadas, como explicita Marcuschi (2010, p. 25) em sua definição de oralidade como "prática social interativa para fins comunicativos" e que "se apresenta sob variadas formas de gêneros textuais fundidos na realidade sonora: ela vai desde a realização mais informal à mais formal nos mais variados contextos de uso".

Pode-se concluir que os dois dicionários abordam as diferenças entre o oral e o escrito e desenvolvem uma história resumida da evolução do tratamento do oral na Didática das Línguas e Culturas. O de Cuq (2003), publicado mais recentemente, aprofunda esse panorama histórico com dados suplementares sobre a abordagem comunicativa tais como as noções de ato de fala e dos gêneros orais, as novas pesquisas sobre a intercompreensão e o trabalho com os jogos de papéis. Pode-se constatar, em Galisson e Coste (1976), a presença dos elementos extralinguísticos importantes e pertinentes, tais como: a entonação, as mímicas, os gestos na definição da noção em questão, não citados no DDFLS. Por outro lado, Cuq (2003) só trata das 
questões de entonação e ritmo no verbete “prosódia". É preciso sublinhar que nenhum dos dois dicionários aborda a questão da oralidade pelo viés das pesquisas que apreendem a linguagem como interação verbal incluindo as relações dialéticas entre intersubjetividade e prática social em suas problemáticas, ou seja, em sua perspectiva discursiva.

\section{Considerações Finais}

Dentro do ensino de língua estrangeira, na Didática das Línguas e Culturas ou na Linguística Aplicada é preciso levar em conta as diferentes modalidades de discursos orais e como trabalhá-los em sala de aula. Pois como afirma Schneuwly e Dolz (2010, p. 117):

Há pouca coisa em comum entre a performance de um orador e a conversa cotidiana; entre uma tomada de turno num debate formal e a discussão num grupo de trabalho; entre uma aula dada e uma explicação numa situação de interação imediata; entre a narração de um conto em sala de aula e o relato de uma aventura no pátio do recreio. Os meios linguísticos diferem fundamentalmente; as estruturas sintáticas e textuais são diferentes; a utilização da voz, sempre presente, também se faz de maneira diversa; e igualmente a relação com a escrita é, em cada situação, específica.

Além desse aspecto mais prático de sala de aula e do material didático, mostra-se ainda essencial, no ensino de línguas hoje, trazer, às pesquisas da área, a vertente discursiva, que coloca o sujeito e suas relações com a sociedade e com o seu inconsciente no centro das preocupações didáticas. O professor, o formador e o pesquisador devem investigar e propor a reflexão sobre o lugar que o aprendiz pode ocupar em cada tipo de discurso, sobre as funções que pode assumir e sobre as regras que devem ser consideradas em cada gênero oral. Ainda que, Bakhtin e Volichínov já afirmassem, em 1929, que os "Gêneros mais livres e criativos de comunicação discursiva oral: os gêneros das conversas de salão sobre temas do cotidiano, sociais, estéticos e similares, os gêneros de conversas à mesa, das conversas íntimo-amistosas, íntimo-familiares, etc.” (BAKHTIN, 2010, p. 283) devessem ser estudados, usados e reformulados, pode-se observar que, até hoje, no ensino de língua estrangeira, existe a necessidade de formar nossos professores para a utilização desses instrumentos com desenvoltura. 
$\mathrm{Na}$ prática do ensino de língua estrangeira, acreditou-se que a substituição de estruturas por atos de fala seria suficiente para garantir o desenvolvimento da linguagem oral, mas, é preciso, "ao menos, ter algo a dizer, para quem dizer e razões para fazê-lo" (CORACINI, 1999, p. 121), proposta de Coracini corroborada por Bakhtin no sentido de dar prioridade à "intenção discursiva do falante, com toda a sua individualidade e subjetividade" (BAKHTIN, 2010, p. 283).

A disciplina tem muito a ganhar considerando os estudos sobre a intersubjetividade e a perspectiva dialógica. Os dois dicionários analisados não levaram em conta esses aspectos. O primeiro, DDL (GALISSON; COSTE, 1976), devido talvez à época de sua publicação, durante a qual essas pesquisas ainda não haviam sido desenvolvidas de forma suficientemente aprofundada para serem aplicadas na Didática de Línguas e Culturas. O segundo, o DDFLES, apesar de mais recente (CUQ, 2003), parece que se restringiu a pesquisas em voga na França como a intercompreensão e o tratamento automático das línguas.

No Brasil, as pesquisas sobre a perspectiva discursiva têm se desenvolvido de forma intensa tanto no aprendizado de língua materna quanto de língua estrangeira, estando presentes até mesmo nos PCNs. É imprescindível, no ensino de línguas estrangeiras, priorizarmos a necessidade de se levar em conta nossas próprias experiências, como afirma Coracini (2003, p. 154):

Falar ou escrever, ler ou ouvir em qualquer língua significa produzir sentido e isso só se dá a partir da história de cada um, das vozes (experiências, reflexões, outras leituras, discussões, valores, crenças) que pouco a pouco, vão construindo e alterando a subjetividade.

O ensino-aprendizagem de línguas estrangeiras deve se libertar dos materiais prontos e da ditadura de formas de ensinar que não se adaptam às nossas vozes e às nossas experiências de vida, ou seja, é preciso chegar ao outro construindo as nossas identidades sem, no entanto, ser dominado por ele. 


\section{Referências}

ARTAUD, A. Le théâtre et son double. «La mise en scène de la métaphysique », Cuvres complètes. Paris : Gallimard, 1964. t. IV.

BAKHTIN, M. Estética da criação verbal. 5. ed. Tradução Paulo Bezerra. São Paulo: Martins Fontes, 2010 [1979].

BAKHTIN, M.; VOLOCHÍNOV, V. N. Marxismo e filosofia da linguagem. 14. ed. Tradução de Michel Lahud e Yara Frateschi Vieira. São Paulo: HUCITEC, 2010 [1929].

CORACINI, M. J. (Org.). Interpretação, autoria e legitimação do livro didático. Campinas: Pontes, 1999.

CORACINI, M. J. Identidade e discurso. Campinas: Editora da Unicamp, 2003.

CORACINI, M. J.; GHIRALDELO, C. M. Nas malhas do discurso: memória, imaginário e subjetividade. Campinas: Pontes, 2011

CUQ, J.-P. (Org.). Dictionnaire de didactique du français. Langue étrangère et seconde. Paris : CLE International/ASDIFLE, 2003.

CUQ, J.-P. Cours de didactique du français langue étrangère et seconde. Grenoble : Presses Universitaires de Grenoble. 2005.

GALISSON, R.; COSTE, D. (Orgs.). Dictionnaire de didactique des langues. Hachette, Paris, 1976.

GALISSON, R.; PUREN C. La formation en question. Paris : CLE International, Didactique des langues étrangères, 1999.

MARCUSCHI, L. A. Produção textual, análise de gêneros e compreensão. São Paulo: Parábola Editorial, 2008.

MARCUSCHI, L. A. Da fala para a escrita: atividades de retextualização. 10. ed. São Paulo: Cortez, 2010.

MASSARO, P. O silêncio e a voz do texto teatral em Francês Lingua Estrangeira. 2007. Tese (Doutorado em Língua e Literatura Francesa) - Universidade 
de São Paulo, São Paulo. 2007. Disponível em: <http://

www.teses.usp.br/teses/disponiveis/8/8146/tde-03092007-132129/ptbr.php>.

MESCHONNIC, H. Critique du rythme. Lagrasse : Verdier, 1982.

MESCHONNIC, H. Poétique du traduire. Lagrasse : Verdier, 1999.

MESCHONNIC, H. Poética do tradųir. Tradução Jerusa Pires Ferreira e Suely Fenrich. São Paulo: Perspectiva, 2010.

MOITA LOPES, L. P. (Org.). Por uma linguística INDISCIPLINAR. São Paulo: Parábola Editorial, 2006.

PIETRARÓIA, C. Les chemins du lecteur. In: Synergies Brésil, Revue de didactologies des langues - Cultures, Florianópolis, n. 1, 2000.

REIS, M. G. M. O texto teatral e o jogo dramático no ensino de francês língua estrangeira. 2008. Tese (Doutorado em Língua e Literatura Francesa) Universidade de São Paulo, São Paulo. 2008. Disponível em: <http:/ / www.teses.usp.br/teses/disponiveis/8/8146/tde-02122008-171004/ptbr.php>.

SCHENEUWLY, B.; DOLZ, J. Gêneros orais e escritos na sala de aula. 2. ed. Tradução e organização: Roxane Rojo e Glaís Sales Cordeiro. São Paulo: Mercado de Letras, 2010. 